\title{
SMEC3D-Mobi: um jogo para Estimulação Cognitiva em plataforma móvel
}

\author{
Ian Nantes Linares Bastos \\ Departamento de Ciência da Computação \\ Universidade do Estado do Rio de Janeiro \\ Rio de Janeiro, Brasil \\ ianbastos98@hotmail.com
}

Rosa Maria E. Moreira da Costa Departamento de Ciência da Computação Universidade do Estado do Rio de Janeiro Rio de Janeiro, Brasil rcosta@ime.uerj.br

\author{
Pedro Ivo de Araujo Marques \\ Departamento de Ciência da Computação \\ Universidade do Estado do Rio de Janeiro \\ Rio de Janeiro, Brasil \\ pedroivomarques@hotmail.com.br
}

\author{
Vera Maria B. Werneck \\ Departamento de Ciência da Computação \\ Universidade do Estado do Rio de Janeiro \\ Rio de Janeiro, Brasil \\ vera@ime.uerj.br
}

\begin{abstract}
Considering the evolution and the spread of mobile equipment, and the expansion of networks, old software can no longer attract the attention of its users, especially when they are on desktop platforms. This paper proposes to describe some aspects related to the migration process of a multiagent system with tridimensional interface, which works on desktop, to the mobile platform, aiming to be used in smartphone support equipment for three-dimensional visualization, in cognitive stimulation processes.
\end{abstract}

Keywords-Cognitive Stimulation, Virtual Reality, Software Reengineering

Resumo - Considerando a evolução e a disseminação de equipamentos móveis e a expansão de redes, os programas antigos não consegue mais atrair a atenção de seus usuários, principalmente quando estão em plataformas desktop. Este artigo propõe descrever alguns aspectos relacionados ao processo de migração de um sistema multiagentes com interface tridimensional, que funciona em desktop, para a plataforma móvel, visando ser utilizado em equipamentos de suporte a smartphones para visualização tridimensional, em processos de estimulação cognitiva.

Palavras-chave - Estimulação Cognitiva, Realidade Virtual, Reengenharia de Software

\section{INTRODUÇÃO}

Atualmente, a evolução dos dispositivos móveis e das redes abre novas perspectivas de acesso a informações e aplicativos para diferentes grupos da população. Esses dispositivos permeiam muitas áreas e apoiam a realização de muitas atividades de modo acessível e rápido. A evolução da tecnologia móvel incentiva o crescimento da oferta de aplicativos. Entretanto, vários programas que funcionam em ambiente desktop, não podem ser usados diretamente em dispositivos móveis. Nesse caso, precisamos estabelecer estratégias de reimplementação desses sistemas, visando ampliar suas capacidades de acesso e disseminação.

Em geral, várias estratégias de migração e reengenharia são propostas e adotadas, como relatado em Ribas et al.[1], Cagnin et al. [2] e Rather\&Vivek [3]. Entretanto, não há um modelo que contemple todos os tipos de modificações a serem realizadas nos sistemas, sejam em relação a suas funcionalidades, ou plataformas.

Considerando as áreas de educação e treinamento, os aplicativos podem ajudar no processo de aprendizagem de novos conceitos de uma maneira mais lúdica, ou podem apoiar processos de atenção e memória de pessoas que sofreram doenças, ou acidentes, com sequelas cognitivas. Ambientes que estimulam a ludicidade tornam o processo de aprendizagem mais motivador, engajando o usuário em atividades especificas de estimulação de funções cognitivas básicas, como atenção e memória [4].

Neste contexto, foi desenvolvido jogo SMEC3D [5], voltado para apoiar a estimulação cognitiva, explorando uma plataforma multiagentes, com visualização de cenas tridimensionais (3D) e estratégias de gamificação. Entretanto, a linguagem adotada na construção do SMEC3D apresenta limitações gráficas, que tornam as cenas pouco reais.

$\mathrm{O}$ avanço dos motores gráficos, associado à difusão cada vez mais crescente do uso de equipamentos móveis para proporcionar imersão, estimulou o processo de reimplementação do SMEC3D. Nesse sentido, o objetivo deste artigo é apresentar a proposta de revisão e reimplementação do sistema SMEC3D, considerando a adoção da ferramenta UNITY [6] para o desenvolvimento de cenas 3D, com visualização em suporte para smartphones.

A próxima seção descreve o ambiente SMEC3D, destacando suas funcionalidades e arquitetura. Na seção III são apresentados alguns trabalhos, que abordam a questão da migração e reengenharia de sistemas, destacando alguns aspectos técnicos. A seção IV apresenta algumas características do SMEC3D-Mobi, que adota a mesma estrutura de multiagentes do sistema anterior, mas tem uma nova interface e novos parâmetros considerando a plataforma Andróide. Na Conclusão são destacados os trabalhos futuros, seguida pelas referências.

\section{O SMEC3D}

O SMEC3D é um jogo, com características de "serious games", para estimular as funções cognitivas de memória, atenção e concentração de pessoas com diferentes tipos de distúrbios neuropsiquiátricos. Em geral, os Serious Games 
exploram atividades, que ajudam na recuperação de funções cognitivas ou motoras e na aprendizagem de novos conceitos [5].

Nos últimos anos, nosso grupo de pesquisa testou algumas metodologias para integrar multiagentes e ambientes tridimensionais [7], [8]. No caso do SMEC3D, para suportar todas as fases de modelagem e implementação dos agentes, um catálogo léxico foi especificado para registrar e documentar os requisitos. Foram definidos quatro agentes, que são responsáveis pelo planejamento da assistência ao paciente, pelo controle das interações no jogo, pela análise do desempenho do usuário e pela alteração do ambiente 3D.

Para a implementação dos agentes e do ambiente 3D, foram pesquisadas várias linguagens e frameworks, verificando-se a compatibilidade tecnológica entre eles. Entre os artefatos estudados, destacaram-se: X3D [9], JAVA [10] e o framework JADE [11]. Para integrar esses três aplicativos foi usada a Plataforma NetBeans [12]. O comportamento dos agentes foi baseado em um modelo simplificado de BDI (Believes, Desires ans Intentions) [13], que cria relações entre os agentes.

O número de agentes foi definido a partir de entrevista com um especialista da área de aplicação. A partir dos resultados dos estudos sobre diferentes metodologias de modelagem de sistemas multiagentes [7], [8], foi adotado o framework orientado a metas i* (i-star) [14]. Neste modelo é possível descrever as relações de dependências intencionais entre atores estratégicos e suas justificativas. A Figura 1 apresenta o Diagrama de Dependência Estratégica - SD modelado na estrutura $i *$. O diagrama SD foi usado para identificar os atores (usuários e agentes), as tarefas e os recursos necessários para processar as tarefas. O SD define o dependências de rede entre os atores e suporta a identificação de agentes, usuários e seus relacionamentos.

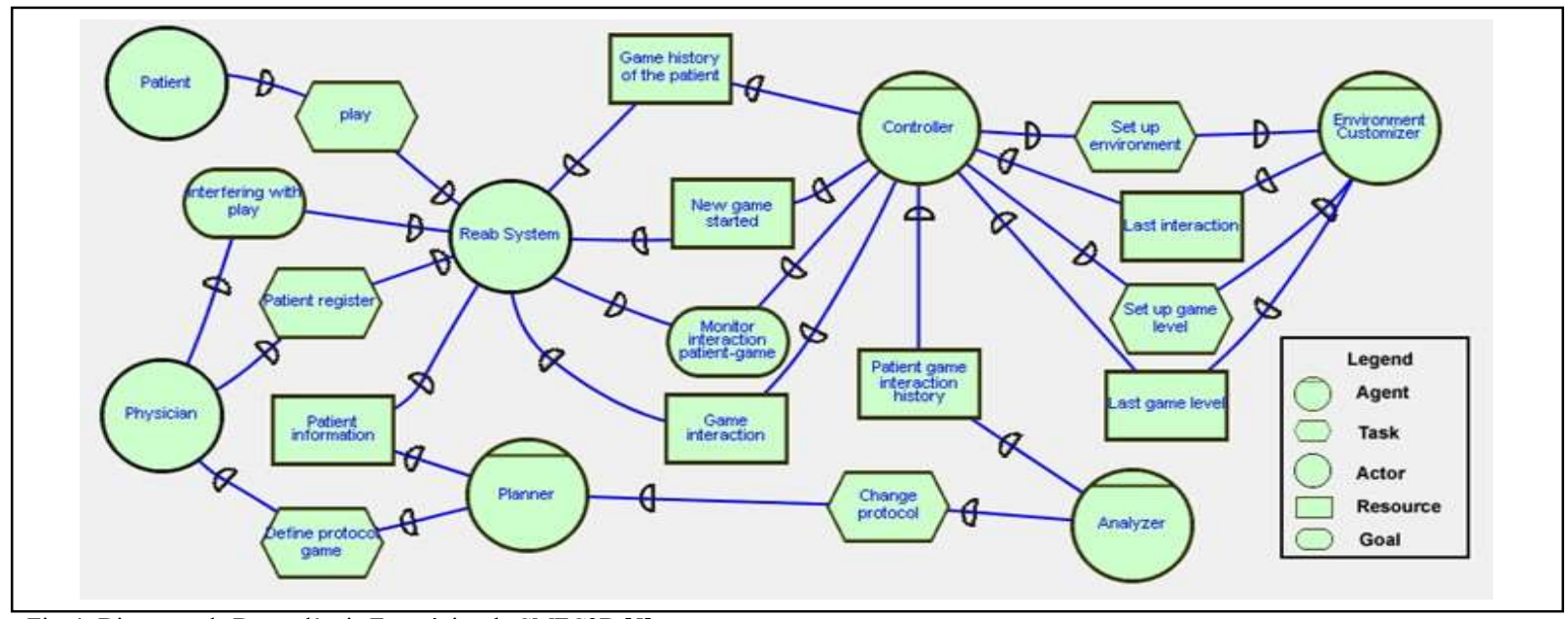

Fig. 1. Diagrama de Dependência Estratégica do SMEC3D [5].

O jogo propõe atividades em dois espaços: sala e cozinha. A sala tem prateleiras de livros, com bolas ou cubos de cores diferentes, que são mostrados aleatoriamente em uma mesa e o paciente deve escolher o que é similar nas prateleiras. $\mathrm{O}$ segundo espaço é uma cozinha, onde são mostrados itens relacionados a alimentação e decoração e o usuário deve procurar o similar.

Os agentes monitoram o tempo e as interações do usuário com o ambiente. Eles controlam as respostas certas e erradas e combinam esses dados com as informações de protocolos, que estão armazenados no sistema. O terapeuta é responsável por registrar os dados pessoais do paciente no sistema e a partir deles, um agente associa um protocolo previamente armazenado.

Este jogo tem oito níveis de dificuldade. Em cada nível, o paciente terá 10 rodadas e deve ter pelo menos, sete respostas corretas para prosseguir para o próximo nível. Se os itens mínimos não forem atingidos, o paciente permanecerá no nível atual. Isso pode ocorrer até quatro vezes. Caso contrário, a sessão terminará.

A Figura 2 apresenta uma imagem da primeira fase do SMEC3D.

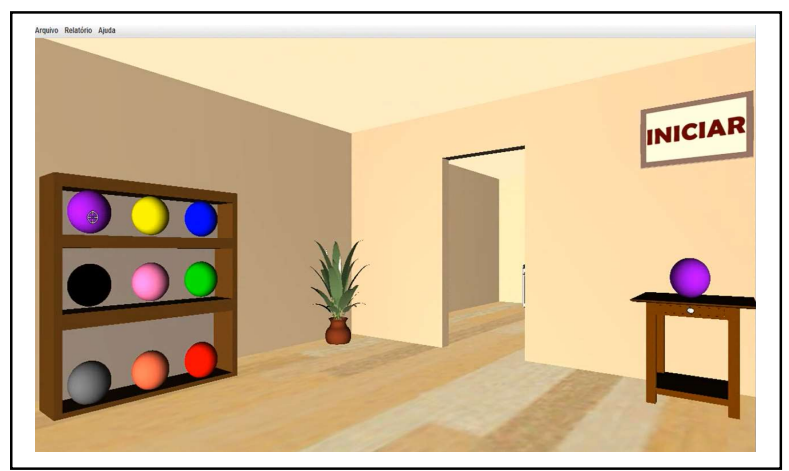

Fig. 2. Imagem de uma das fases do jogo na primeira cena [5].

O SMEC3D foi desenvolvido a partir de estudos que destacaram tecnologias, que permanecem atuais e eficientes. Entretanto, sua interface gráfica encontra-se desatualizada em relação às expectativas dos usuários, que frequentam salas de cinema 3D e mesmo, já possuem TVs com tecnologias imersivas em suas casas.

Herpich e Tarouco [15] observaram que a crescente inserção de ambientes tridimensionais em contextos educacionais aumenta $\mathrm{o}$ engajamento dos usuários no 
aprendizado de novos conceitos e amplia as possibilidades de comunicação e cooperação.

Logo, torna-se essencial que os ambientes 3D ofereçam novas perspectivas de utilização para seus usuários e que promovam o engajamento, que por sua vez, estimula os processos de apreensão de conceitos e práticas.

\section{TRABALHOS CORRELATOS}

$\mathrm{Na}$ busca de estratégias e metodologias de apoio a migração de sistemas, estudamos algumas propostas.

De acordo com Canto [16], o que afeta o processo de migração é a carência, ou a ausência, de documentação técnica dos sistemas legados. Para os autores, em muitos casos, o processo de migração de um sistema para outra plataforma considera uma "tradução" do código. Dessa forma, não haveria necessidade de re-especificar o funcionamento do sistema em uma modelagem de alto nível, necessitando apenas de engenharia reversa e considerar que se trata de um novo sistema. Nesta visão, esse processo deveria se apoiar em especificações técnicas e não, no código-fonte original. Ele ressalta que o melhor seria que o desenvolvedor nem tivesse contato com o código fonte original.

Segundo Cagnin [2] o desenvolvimento de novas tecnologias tem gerado a necessidade de evolução de sistemas legados. Diante desse quadro, variados métodos de reengenharia têm sido propostos na literatura, mas poucas ferramentas computacionais têm sido propostas para apoiar esse processo. Visando preencher essa lacuna, Cagnin [2] propôs um framework para realizar a engenharia reversa do sistema legado, com o apoio de linguagem de padrões de análise, assim como, dos recursos de testes associados.

Amorim et al. [17] apresentaram o processo de remodelação de um software, o SACAR-Web, usando algumas estratégias de desenvolvimento de software iterativo incremental, considerando cada parte do sistema como uma iteração.

Com o avanço das redes e dos equipamentos móveis, a sociedade cria novas demandas de modelos de interface e de interação. A partir dos estudos preliminares na literatura que trata de migração e reengenharia de softwares, observamos que as razões para realizar uma reimplementação de software apresenta várias motivações. No caso específico do SMEC3D, a linguagem utilizada para o desenvolvimento das cenas tinha limitações em termos de iluminação e realismo. E também, não apresentava possibilidades de utilização em equipamentos imersivos por causa da estrategia de interação adotada, com o uso de mouse.

Em relação ao modelo de migração, optou-se por adotar uma prática mais simples, considerando aspectos de revisão dos documentos e modelos de agentes, das estratégias de comunicação entre os agentes, mas focando, principalmente, na reformulação do módulo de realidade virtual, seguindo a proposta de Canto [16], de nem mesmo, ter acesso ao códigofonte desse módulo. Mas, ao mesmo tempo, adotando uma visão iterativa, com ciclos evolutivos, de acordo com a visão de Amorim et al. [17].

A seção a seguir apresenta os resultados obtidos até então, nesse processo de reimplementação do SMEC3D.

\section{RESUltados DA PROPOSTA DE REIMPLEMENTAÇÃO DO SMEC3D}

A partir das percepções sobre os temas envolvidos na tarefa de reimplementar o SMEC3D, considerando as plataformas móveis, a Figura 3 apresenta um modelo iterativo simplificado das etapas adotadas nesse processo e o ambiente passa, então, a se chamar SMEC3D-Mobi.

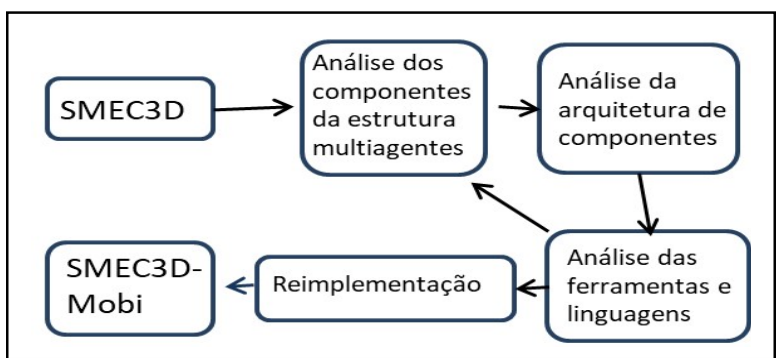

Fig 3. Modelo de processo de reimplementação do SMEC3D

Iniciamos o estudo avaliando a estrutura de agentes adotada no SMEC3D. Apesar de ser uma metodologia de modelagem com um nível de complexidade bastante significativo, optamos por enquanto, por manter os agentes e os modelos de comunicação adotados, como visto na Figura 1 .

A arquitetura de componentes sofreu algumas modificações considerando, principalmente, o motor gráfico de desenvolvimento do módulo de realidade virtual (Figura 4).

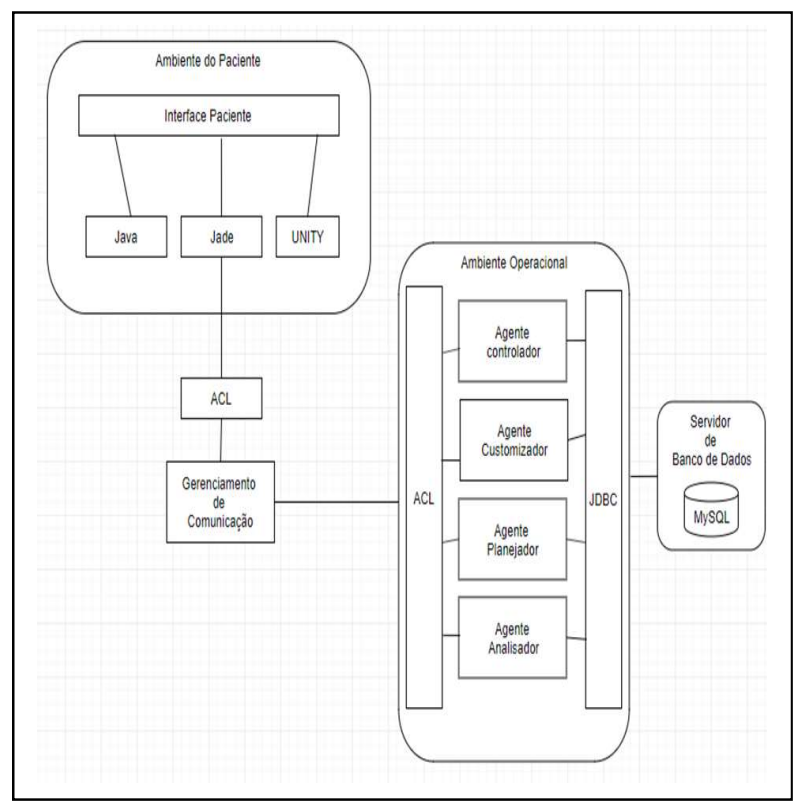

Fig 4. Arquitetura dos componentes do SMEC3-Mobi

O banco de dados adotado é o MySQL [18], que irá guardar os dados cadastrais de usuários e terapeutas, assim como, os resultados da navegação dos usuários. Ele está conectado ao NetBeansIDE [12], que por sua vez, armazena o back-end e o front-end da plataforma. O NetBeansIDE conecta o JADE [11] com o Banco de Dados e sua IDE está servindo para conectar também o front-end. 
O motor gráfico é o UNITY [6], onde os ambientes 3D estão sendo criados. Neste caso, a nossa proposta visa criar cenas menos artificias, com iluminação e composição da cena mais reais. Para evitar os problemas associados às constantes atualizações do UNITY, vamos adotar o UNITY Hub, que é um aplicativo recente, incluído no UNITY, que apoia o gerenciamento dos projetos e instalações da ferramenta.

Os agentes continuam a ser desenvolvidos no framework JADE (Java Agent Development Framework) [11]. Os agentes a princípio terão as mesmas funcionalidades daqueles implementados no SMEC3D, mas de acordo com a metodologia adotada, eles poderão sofrer alterações em alguma das iterações do processo de desenvolvimento.

Nesse contexto, estamos considerando a possibilidade de utilização de outras ferramentas, como o React [19], que é uma biblioteca JavaScript de código aberto, para criar interfaces de usuário.

A plataforma adotada na versão do novo SMEC3D-Mobi é a móvel, iniciando-se pela plataforma Andróide.

Uma imagem da primeira versão da cena do primeiro nível do jogo pode ser observada na Figura 5.

Este ambiente poderá ser visualizado em um suporte para visualização $3 \mathrm{D}$ a partir de smartphones. A interação dos usuários com a cena será realizada com a técnica de Raycasting para escolher o objeto correto na cena [20]. Nesta técnica, um cursor fica em cena e pode ser movimentado a partir dos movimentos da cabeça do usuário. Para selecionar um objeto basta posicionar esse cursor sobre o objeto desejado e aguardar alguns segundos.

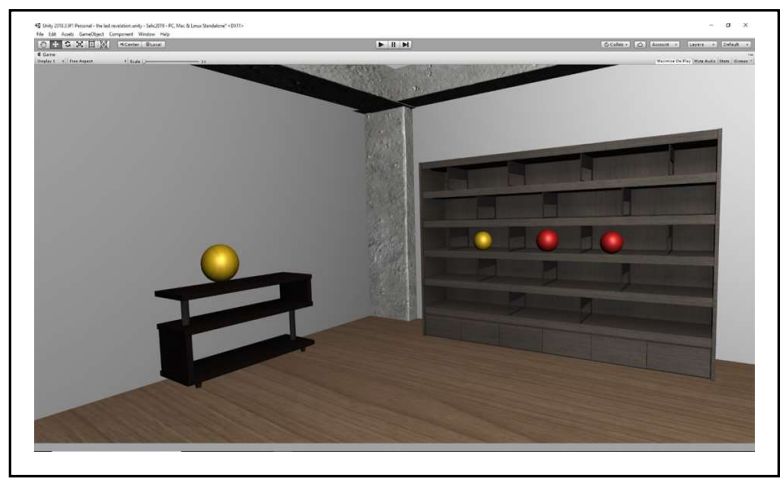

Fig 5. Imagem da cena do primeiro ambiente do SMEC3D-Mobi

\section{CONCLUSÃO}

Este artigo apresentou uma proposta e resultados iniciais da reimplementação de um sistema voltado para a Estimulação Cognitiva de pessoas com déficits de atenção e memória. Para tanto, foram apresentadas as características do sistema original, alguns exemplos de trabalhos que discutem aspectos de migração e reengenharia de sistemas legados. A proposta considerou alguns aspectos dessas abordagens para propor um modelo que dividiu o problema em módulos, que podem ser estudados/modificados, e essas modificações podem ser distribuídas e consideradas em outras etapas do desenvolvimento do sistema.

Resultados preliminares apontam para a realização de poucas modificações do módulo dos agentes, que controlam todo o sistema. Na etapa que estuda a arquitetura, trocou-se o motor gráfico, para o UNITY, que cria cenas mais realistas e implementa o processo de navegação e interação do usuário nas cenas de maneira mais eficiente.

A possibilidade de uso desse sistema modificado em equipamentos imersivos, com o apoio de smartphones, abre perspectivas de aumento de interesse para seus usuários.

Como próximos passos, temos o estudo mais aprofundado de técnicas de modelagem e implementação de ambientes 3D, o estudo do framework JADE, para a reimplementação dos agentes e novas entrevistas com especialistas da área da saúde, para atender requisitos, que não foram contemplados na primeira versão: protocolos adaptáveis a mais tipos de deficiências e relatórios com informações mais completas sobre o desempenho dos usuários.

Este projeto permite que alunos participantes de programas de Iniciação Científica tenham contato com tecnologias de ponta, interajam com profissionais de outras áreas e se sintam motivados em termos de difusão para a sociedade, já que esse sistema poderá ser utilizado em situações terapêuticas, na área da saúde mental.

\section{AGRADECIMENTOS}

Este estudo é parcialmente apoiado pelo CNPq e pela UERJ, por meio de bolsas de Iniciação Científica.

\section{REFERÊNCIAS}

[1] M. Ribas, A. S.Lima, J. N.de Souza, F. R. de Carvalho Sousa, and G. Fenner, "Tomada de decisão multicritério na migração de aplicativos para ambientes de nuvem do tipo software as a service". Revista Brasileira de Administração Científica, vol 5, n.2, 2014, pp. 83-94.

[2] M.Cagnin, J. Maldonado, A. Chain, "Reuso na Atividade de Teste para Reduzir Custo e Esforço de VV\&T no Desenvolvimento e na Reengenharia de Software”. In: XVIII Simpósio Brasileiro de Engenharia de Software (SBES), 2004, pp.71-84.

[3] Manzoor Ahmad Rather, and Mr Vivek Bhatnagar. "Study of Software Development Using Software Re-Engineering." , International Journal of Engineering Trends and Applications (IJETA) - Vol.3 n. 2, 2016 pp. 53-54

[4] R. M. Moraes, L. S.Machado, F.L.S. Nunes, and R. M. E. M. Costa, "Serious Games and Virtual Reality for Education, Training and Health". In: Maria Manuela Cruz-Cunha. (Org.). Handbook of Research on Serious Games as Educational, Business and Research Tools. 1ed.Pennsylvania: IGI Global, 2012, v. 1, pp. 315-336.

[5] Priscilla F. de Abreu, Vera Maria B. Werneck, Luis Alfredo V. de Carvalho, and Rosa M. E. M. Costa. "Employing Multi-agents in 3-D Game for Cognitive Stimulation”. In: XIII Symposium on Virtual Reality (SVR), Uberlandia. 2011, v. 1. pp. 73-83.

[6] UNITY, In: https://unity3d.com/pt/unity, consultada em agosto/2019.

[7] Rosa M. E. M. Costa, Israel Mendonça, and Diogo Souza, "Exploring the intelligent agents for controlling user navigation in 3D games for cognitive stimulation". In: $8^{\text {th }}$ International Conference on Disability, Virtual Reality and Associated Technologies, Viña del Mar. Reading: University of Reading, v. 1, 2010. pp. 1-6.

[8] Vitor M. F Ferreira, Julio C. C. Carvalho, V. M. B.Werneck, and Rosa M. E. M. da Costa, "Developing an educational medical game using AgilePASSI multi-agent methodology". In: IEEE 28th International Symposium on Computer-Based Medical Systems, 2015. pp. 298303.

[9] Brutzman, D. e Daly, L.. "X3D: 3D Graphics for Web Authors". Morgan Kaufmann Publishers, 2007.

[10] JAVA. In: http://www.java.com/pt_BR/, acessado em Agosto 2019.

[11] JADE. In: http://jade.tilab.com/, acessado em Agosto 2019.

[12] Netbeans. In: http://netbeans.org/, acessado em Agosto 2019. 
[13] J. M. Bradshaw, "An introduction to software agents", In: Bradshaw, J. M. Ed. Software Agents. Massachusetts: MIT Press, 1997.

[14] E.Yu, "Modeling Strategic Relationships for Process Reengineering", In: Yu E, Giorgini P, Maiden N, editors. Mylopoulos. J. Social Modeling Engineering, 2011.

[15] F. Herpich, and L. M. R. Tarouco, "Engajamento de usuários em mundos virtuais: Uma análise teórica-prátic." RENOTE, vol 14, n.1, 2016.

[16] F. H. Canto, "Migração de software: considerações e experiências.", In: Workshop de Tecnologia de Informação e Comunicação das Instituições Federais de Ensino Superior. Gramado, 2016.
[17] T. A.Amorim, et al. "Remodelagem do software sacar-web usando técnicas de engenharia reversa e reengeharia de software.", In: Congresso Brasileiro de Informática em Saúde-CBIS, 2006.

[18] MySQL. In: http://www.mysql.com, acessado em julho de 2019.

[19] REACT, In: https://pt-br.reactjs.org/, acessado em agosto de 2019.

[20] D. P. Costa, P. Sampaio, and V. F. Martins, "Gesture interaction metaphors within 3D environments: Revisiting the literature.", In: XLIII Latin American Computer Conference (CLEI). IEEE, 2017. 